\title{
Effect on breastfeeding practices of providing in-home lactation support to vulnerable women through the Canada Prenatal Nutrition Program: protocol for a pre/post intervention study
}

Alison Mildon ${ }^{1} \mathbb{D}$, Jane Francis ${ }^{1,2} \mathbb{D}$, Stacia Stewart ${ }^{3}$, Bronwyn Underhill ${ }^{3}$, Yi Man $\mathrm{Ng}^{3}$, Elle Richards ${ }^{4,5}$, Christina Rousseau ${ }^{4}$, Erica Di Ruggiero ${ }^{6}$, Cindy-Lee Dennis ${ }^{7}$, Deborah L. O'Connor ${ }^{1,2+}$ (D) and Daniel W. Sellen ${ }^{1,6,8^{*}+}$ (D)

\begin{abstract}
Background: Only one-third of Canadian infants are exclusively breastfed for the first 6 months of life as recommended. Skilled lactation support in the early postpartum period is one strategy for improving breastfeeding outcomes by building breastfeeding self-efficacy and resolving difficulties. Access to such support is limited among vulnerable women, including those who are new immigrants, low income, under-educated, young or single. The Canada Prenatal Nutrition Program (CPNP) aims to improve birth and breastfeeding outcomes among vulnerable women, but currently lacks a formal framework for providing postpartum lactation support.

Methods: This pre/post intervention study will examine the effect on breastfeeding outcomes of an evidencebased in-home lactation support intervention provided through the CPNP. We will enrol 210 pregnant women who intend to breastfeed and are registered CPNP clients at two sites in Toronto, Canada. During the intervention phase, postpartum home visits by International Board Certified Lactation Consultants (IBCLCS) will be pro-actively offered to registered clients of the two sites. Double-electric breast pumps will also be provided to those who meet specific criteria. Infant feeding data will be collected prospectively at seven time points from 2 weeks to 6 months postpartum. Descriptive and regression analyses will be conducted to measure intervention effects. The primary outcome is exclusive breastfeeding at 4 months postpartum. Secondary outcomes include the duration of any and exclusive breastfeeding, timing of introduction of breastmilk substitutes and timing of introduction of solid foods. Breastfeeding self-efficacy will be assessed prenatally and at 2 weeks and 2 months postpartum. Other measures include maternal socio-demographics, infant feeding intentions, maternal depression and anxiety, and household food insecurity. Monitoring data will be used to assess the reach, uptake and fidelity of intervention delivery.
\end{abstract}

\footnotetext{
* Correspondence: dan.sellen@utoronto.ca

${ }^{\dagger}$ Deborah L. O'Connor and Daniel W. Sellen are co-senior authors.

${ }^{1}$ Nutritional Sciences, Temerty Faculty of Medicine, University of Toronto, Toronto, Ontario, Canada

${ }^{6}$ Dalla Lana School of Public Health, University of Toronto, Toronto, Ontario,

Canada

Full list of author information is available at the end of the article
}

(c) The Author(s). 2021 Open Access This article is licensed under a Creative Commons Attribution 4.0 International License, which permits use, sharing, adaptation, distribution and reproduction in any medium or format, as long as you give appropriate credit to the original author(s) and the source, provide a link to the Creative Commons licence, and indicate if changes were made. The images or other third party material in this article are included in the article's Creative Commons licence, unless indicated otherwise in a credit line to the material. If material is not included in the article's Creative Commons licence and your intended use is not permitted by statutory regulation or exceeds the permitted use, you will need to obtain permission directly from the copyright holder. To view a copy of this licence, visit http://creativecommons.org/licenses/by/4.0/. The Creative Commons Public Domain Dedication waiver (http://creativecommons.org/publicdomain/zero/1.0/) applies to the data made available in this article, unless otherwise stated in a credit line to the data. 


\begin{abstract}
Discussion: Increasing access to skilled lactation support through the CPNP may be an effective means of improving breastfeeding practices among vulnerable women and thereby enhancing health and development outcomes for their infants. This pre/post intervention study will contribute evidence on both the effectiveness and feasibility of this approach, in order to guide the development and further testing of appropriate models of integrating lactation support into the CPNP.
\end{abstract}

Trial registration: ClinicalTrials.gov (NCT03589963) registered July 18, 2018.

Keywords: Exclusive breastfeeding, Vulnerable women, Lactation support, Canada Prenatal Nutrition Program

\section{Background}

Breastmilk is a unique food tailored to meet the nutritional, immunological and developmental needs of human infants [1]. The health benefits of breastfeeding are realized in a dose-response relationship to the degree of exclusive breastfeeding, particularly in early infancy [1]. The World Health Organization and Health Canada recommend that all infants be exclusively breastfed (i.e. receive only breastmilk and essential vitamins, minerals and medicines) for the first 6 months of life [2,3]. However, achievement of this goal remains elusive. Globally, only $42 \%$ of infants under 6 months of age are exclusively breastfed [4]. National data show that $90 \%$ of Canadian mothers initiate breastfeeding, but nearly half discontinue within the first 6 months and only 32\% exclusively breastfeed for 6 months [5]. Exclusive breastfeeding is compromised by a variety of practices, particularly formula supplementation and early introduction of solids [6, 7]. Exclusive breastfeeding duration is lower among vulnerable women in Canada, such as those with lower income and education levels, adolescents, single parents and those living in food insecure households [8-12]. Global systematic review evidence indicates that immigrant women are more likely to initiate and continue breastfeeding than their native-born counterparts, likely reflecting social norms regarding breastfeeding in many countries of origin, but exclusive breastfeeding is a universal challenge [13].

Two key contributors to both early cessation and nonexclusive breastfeeding are the perception of insufficient breastmilk supply and unresolved breastfeeding difficulties [14-17]. Access to skilled lactation support in the early postpartum period can be vital to addressing these concerns and supporting women to achieve their breastfeeding goals [18]. Underlying these concerns is breastfeeding self-efficacy, a construct drawn from Bandura's socialcognitive theory and defined as a woman's confidence in her ability to breastfeed her new baby [19]. Women with high breastfeeding self-efficacy are better able to overcome common challenges and continue breastfeeding [19]. Theory-based postpartum lactation support interventions have been shown to improve breastfeeding self-efficacy, duration and exclusivity $[20,21]$.
Breastfeeding self-efficacy is modifiable through interventions which increase women's experience of four determinants, the most critical of which is performance mastery, or successful achievement of breastfeeding skills [19]. For women who are new to breastfeeding or experiencing difficulties, skilled lactation support in the early postpartum period helps build breastfeeding skills and resolve challenges, directly contributing to performance mastery. Skilled lactation support also contributes to breastfeeding self-efficacy through verbal persuasion (positive, confidence-building messages) and physiological and affective states, through the mitigation of stress, anxiety and pain related to breastfeeding [19]. The fourth determinant of breastfeeding self-efficacy is vicarious learning (observing others successfully breastfeeding), which can be achieved through peer support, media and other forms of role modeling.

Several recent systematic reviews have found evidence of the effectiveness of postpartum skilled lactation support interventions for increasing breastfeeding duration and exclusivity in the first 6 months postpartum [2225]. The authors of a Cochrane review concluded that postpartum lactation support is likely to be more effective when breastfeeding initiation rates are high, and when the support is proactive and predictable with faceto-face contact [24]. However, access to skilled lactation support which follows these best practice guidelines is limited as many services, such as drop-in breastfeeding clinics and telephone hotlines, are provided reactively after problems occur. Vulnerable women in particular may face barriers related to time, transportation and language skills, which make it difficult for them to access these services [26]. Many studies included in the Cochrane review were conducted with low-income women but sub-group analysis by maternal characteristics was not performed [24].

The Canada Prenatal Nutrition Program (CPNP) is an initiative of the federal government implemented through community agencies with the aim of improving birth outcomes and promoting breastfeeding among vulnerable women [27]. Service provision may extend into the postpartum period but this varies between CPNP 
sites, with national evaluation data showing that postpartum women constitute only $12 \%$ of all clients served [28]. Core CPNP services include prenatal breastfeeding promotion and education. Provision of postpartum breastfeeding support is encouraged [29], but there is currently no formal framework or funding for integrating this support within the CPNP. Strengthening this program component may be a means to increase vulnerable women's access to skilled lactation support. An example of this is the infant feeding program implemented with additional charitable funds from The Sprott Foundation by the Parkdale Parents' Primary Prevention Project (5Ps), a CPNP site in Toronto, Canada. Clients of the 5Ps CPNP receive a 'welcome package' of infant care and breastfeeding supplies at the time of delivery, and are offered postpartum in-hospital or in-home visits by International Board Certified Lactation Consultants (IBCLCs) and a double-electric breast pump. A qualitative evaluation found that these lactation services addressed key physical, practical and self-efficacy breastfeeding challenges women encountered, including pain, low milk supply, time pressures and stress [26]. Participants highly valued three specific program elements: the provision of in-home services delivered by skilled providers who were non-judgemental [26]. These findings demonstrate the value of integrating postpartum lactation support with the CPNP, but no data are available to determine the effects of the support provided on breastfeeding practices of 5Ps clients.

Drawing on the evidence from breastfeeding selfefficacy theory, systematic reviews and the 5Ps qualitative evaluation, the aims of the current research are to examine the:

i) effects of providing access to in-home postpartum lactation support on breastfeeding practices among women enrolled in the CPNP at two sites in Toronto; and

ii) feasibility of this delivery model as a means to increase vulnerable women's access to skilled lactation support.

\section{Methods}

\section{Study design and hypothesis}

We will conduct a pre/post intervention study with clients of two CPNP sites in Toronto. Prospectively collected data on infant feeding practices over the first 6 months and breastfeeding self-efficacy scores will be compared between participants who give birth before and after initiation of the lactation support intervention. This quasi-experimental design was chosen as it would not be either ethical or feasible to randomize clients at these community programs targeting vulnerable women to intervention and control groups.
The primary outcome is exclusive breastfeeding at 4 months postpartum. We hypothesize that CPNP clients with access to our lactation support intervention will be more likely to exclusively breastfeed their infants at 4 months postpartum compared with those in the preintervention group, and that this effect will be mediated by higher breastfeeding self-efficacy scores. Secondary outcomes are duration of any breastfeeding; duration of exclusive breastfeeding; timing of introduction of breastmilk substitutes (formula); and timing of introduction of solids. We will also assess the rates of any and exclusive breastfeeding and use of expressed breastmilk at each data collection time point up to 6 months postpartum.

Although 6 months is the recommended duration of exclusive breastfeeding, we chose 4 months for the primary outcome analysis as we anticipate that our intervention will improve exclusive breastfeeding primarily by building breastfeeding skills and addressing difficulties in the early postpartum period. Exclusive breastfeeding is frequently compromised after 4 months through early introduction of solids [6,9], but this practice is less amenable to influence by lactation support provided earlier in the postpartum period.

Monitoring data will be collected and analyzed to determine the reach, uptake and fidelity of the intervention.

\section{Setting}

This research will be embedded within two CPNP sites in Toronto, Ontario: the Great Start Together (GST) program implemented by Parkdale-Queen West Community Health Centre and the Healthy Beginnings (HB) program implemented by The Stop Community Food Centre. These sites were chosen because of their relatively large enrollment size, continuity of service into the postpartum period but limited focus on lactation support, and geographic catchment areas which collectively cover a significant portion of South West Toronto. Both programs operate as weekly drop-in services providing: (1) group workshops; (2) individual support from St. Stephen's Community House settlement staff and Toronto Public Health nurses and dietitians; (3) referrals to other community services, including doula support; (4) CAD $\$ 10$ grocery gift card; (5) snacks during service use; (6) two public transit tokens to attend the program; and (7) on-site childcare during service use. Interpreters attend both programs to enable participation of nonEnglish speaking clients. The nurses deliver group workshops on breastfeeding benefits and techniques approximately every 6 weeks and provide limited individual breastfeeding support to clients on request during the program hours. This support is valuable but has limited reach during the critical early postpartum period as 
many clients do not return to the program for several weeks after giving birth.

Private donations to The Stop Community Food Centre enable the provision of additional supports to $\mathrm{HB}$ clients at the weekly program. These include a hot cooked lunch, CAD $\$ 5$ worth of fresh fruits and vegetables and a food bank hamper typically consisting of $1 \mathrm{~L}$ milk, 4 eggs, 2 cups of rice and 2 cups (or 1 can) of beans. HB's Family Support program provides one-toone support for the most vulnerable clients through home visits.

Monitoring data show that the majority of clients in the programs are newcomers to Canada, defined as arriving within the past 5 years. In 2017, 222 women registered in the GST program, $67 \%$ of whom were newcomers, primarily from China. Chinese interpreter services are provided at the weekly program. From September 2018 to August 2019, 225 women participated in $\mathrm{HB}$, including 134 new clients, 106 of whom were newcomers. In a 2019 internal survey of HB clients $(n=92)$, $55 \%$ reported an annual income below the Statistics Canada household-size-adjusted low-income cut-off [30]. Over $50 \%$ of $\mathrm{HB}$ clients surveyed used interpreter services at the weekly program, which in 2019 included Spanish, Portuguese and American Sign Language.

\section{Sample size}

The sample size estimate is based on the primary outcome. Preliminary data indicated that $53 \%$ of program clients were exclusively breastfeeding at 4 months postpartum. Based on an anticipated increase to $73 \%$ in the post-intervention group, we require 93 women per group in order to achieve 0.80 power with alpha $=0.05$. To allow for $10 \%$ attrition we aim to recruit 105 women per group for a total of 210 study participants. The anticipated attrition rate is based on preliminary evidence of high retention in a separate prospective infant feeding study conducted by our group at another CPNP site.

\section{Recruitment}

Eligibility for the study is defined as registration in GST or HB with a due date within the data collection period; intention to try breastfeeding; and intention to continue living in Toronto with the infant for the first 6 months postpartum. Exclusion criteria are preterm birth before 34 weeks gestation; congenital abnormality or medical condition affecting feeding; and hospitalization of the mother or infant at 2 weeks postpartum.

Recruitment will follow a two-step consent process. New clients of the GST or HB complete a program registration form which includes a yes/no question asking for consent to be contacted about the research. The first author (AM) attends the GST and HB programs weekly and will monitor the registration lists to identify clients in their third trimester of pregnancy. Those who have given permission for contact will be approached to explain the study and obtain informed consent. This contact will take place in person if the client attends programming, or by telephone with provision of the consent form by email.

\section{Intervention}

The core component of the intervention is in-home lactation support delivered by IBCLCs, with provision of breast pumps by the IBCLCs to clients meeting specific criteria (described below) as a secondary component. Enrollment in the research study will not be a precondition for accessing the intervention. This is both for ethical reasons and to allow assessment of the feasibility of this intervention model, including the demand for lactation services by GST and HB clients. The implementation process was formulated in collaboration with the 5Ps Program Coordinator (co-author SS) and infant feeding program staff in order to benefit from their experience and networks. The implementation procedures align as closely as possible with delivery of other services through the GST and HB programs, but clients will be informed that the intervention is being offered as part of an infant feeding research project.

\section{Intervention description}

Clients registered prenatally in either the GST or HB program who give birth during the intervention period and live in the city of Toronto will have access to inhome lactation support from an IBCLC. This service will be offered pro-actively in the early postpartum period, but will be available throughout the first 6 months. A Research Coordinator will oversee intervention delivery, and professional interpreter services (in-person or by telephone) will be used for communication with nonEnglish speaking women.

The Research Coordinator will attend both weekly programs and approach eligible prenatal clients individually to explain the service, provide her contact details, and record the contact information of clients who are interested. Clients will be encouraged to contact the Coordinator if they need lactation support, but the Coordinator will also pro-actively telephone interested clients around the time of birth to offer the IBCLC support and make the arrangements with the IBCLCs as required. IBCLC visits will take place within $48 \mathrm{~h}$ of request by a client, and we anticipate that the majority of initial visits will occur within the first 2 weeks postpartum. Three self-employed IBCLCs already contracted by the 5Ps program have been recruited to deliver the intervention. Private IBCLC services are not covered by provincial health insurance in Ontario, so women who use this resource are typically of higher socio-economic status. The 
5Ps staff previously screened many self-employed IBCLCs to select those who practice with a clientcentred approach and are able to adapt to the circumstances of vulnerable women, such as communicating through interpreters and visiting low-income homes.

The first home visit will last approximately $1.5 \mathrm{~h}$ and include assessment, education and management of the client's lactation concerns. In-person or telephone interpreters will be provided for clients with limited English fluency. The IBCLC conducting the visit will provide her contact information to the client for follow up contact by text, phone or email. Following the first home visit, the Research Coordinator will contact the client to see if they were satisfied with the service and arrange a second visit with the IBCLC if needed. In cases with more complex lactation issues, a third visit may be offered. Follow up visits are expected to be approximately $1 \mathrm{~h}$ in length.

The 5Ps infant feeding program supports include provision of double-electric breast pumps through the IBCLCs, and a majority of clients (70\%) receive this instrumental support. Participants in the 5Ps qualitative evaluation with 46 vulnerable women identified that the breast pumps assisted with building or maintaining breastmilk supply, relieving engorgement, allowing others to feed the baby, and providing breastmilk for infants with latching difficulties [26]. Studies in Canada and other high-income countries report widespread use of breast pumps across socio-demographic groups [6, 31, 32]. Current evidence on the association between breast pump use and breastfeeding outcomes is mixed, and individualized guidance on breast pump use is recommended [33, 34]. Our intervention therefore includes breast pump provision as a secondary component. The IBCLCs will provide a double-electric breast pump (Ameda Finesse ${ }^{\mathrm{Tm}}$ ) to clients who meet the following criteria: (1) infant unable to latch; (2) mother returning to work or school; or (3) high dependence on infant formula.

In addition to the home visits, the IBCLCs will provide three group workshops in each participating CPNP during the intervention phase of the study. At these workshops the IBCLCs will deliver education regarding the onset of lactation in the neonatal period and tips for successfully establishing breastfeeding, as well as explaining the role of IBCLCs and what to expect in a home visit. The workshops will also provide an opportunity for the Research Coordinator to formally introduce and explain the lactation support intervention.

\section{Data collection}

Data will be collected in person or by telephone either by AM or by a Mandarin speaking Research Assistant. Professional interpreter services will be used as required for other non-English speaking participants.
All primary and secondary outcome measures will be assessed through prospective data collection using a standardized and validated Infant Feeding Questionnaire developed by DLO's research group and used in several prior research studies at the Hospital for Sick Children in Toronto [35]. This questionnaire will be administered to study participants at 2 weeks postpartum, and then monthly until 6 months for a total of seven infant feeding data collection points (Table 1). At each time point, participants will be asked to report the average number of daily milk feeds their infant received in the past 2 weeks, divided into feeds at the breast, expressed breastmilk feeds, and formula feeds. Provision of cow's milk, non-milk fluids and vitamin or mineral supplements will also be recorded. Participants who stop breastfeeding will be asked for the last date breastmilk was provided and the main reasons for cessation. If applicable, the date of solids introduction and the name of the first food will be recorded.

We hypothesize that the intervention effects will be mediated through increases in breastfeeding self-efficacy, which will be assessed at 2 weeks and 2 months postpartum using the Breastfeeding Self-Efficacy Scale-Short Form [36]. Additional data collection tools are listed below. The Socio-demographics and 6 Months Postpartum questionnaires were developed for this study, with income-related questions drawn from Statistics Canada's Employment Insurance Coverage Survey [37]. The remaining instruments are validated scales from the literature, all of which have been used previously with vulnerable populations.

a) Maternal Socio-demographics Questionnaire - assesses maternal age, education level, parity, ethnicity and for participants not born in Canada, country of origin and length of time in Canada

b) Infant Feeding Intentions [38] - validated 5-item scale assessing prenatal intention to exclusively breastfeed for 6 months

c) Prenatal Breastfeeding Self-Efficacy Scale [39] - validated 20-item scale; higher prenatal self-efficacy scores are correlated with intention to breastfeed

d) Household Food Security Survey Module of the Canadian Community Health Survey [40] validated 18-item questionnaire; household food insecurity is categorized as marginal, moderate, severe or food secure based on number of affirmative responses

e) Edinburgh Postnatal Depression Scale (EPDS) [41] - validated 10-item scale, widely used in perinatal research and clinical practice to assess maternal depressive symptomatology

f) State-Trait Anxiety Inventory (STAI) [42] validated 40-item scale assessing both current state 
Table 1 Data collection timeline

\begin{tabular}{|c|c|c|c|c|c|c|c|c|}
\hline \multirow[t]{2}{*}{ Study Instrument } & \multirow[t]{2}{*}{ Prenatal } & \multicolumn{7}{|c|}{ Postnatal Data Collection } \\
\hline & & 2 weeks & 1 month & 2 months & 3 months & 4 months & 5 months & 6 months \\
\hline Maternal Socio-demographics Questionnaire & $\sqrt{ }$ & & & & & & & \\
\hline Infant Feeding Intentions Scale & $\sqrt{ }$ & & & & & & & \\
\hline Prenatal Breastfeeding Self-Efficacy Scale & $\sqrt{ }$ & & & & & & & \\
\hline Household Food Security Questionnaire & $\sqrt{ }$ & & & & & & & $\sqrt{ }$ \\
\hline Breastfeeding Self-Efficacy Scale-Short Form & & $\sqrt{ }$ & & $\sqrt{ }$ & & & & \\
\hline Infant Feeding Questionnaire & & $\sqrt{ }$ & $\sqrt{ }$ & $\sqrt{ }$ & $\sqrt{ }$ & $\sqrt{ }$ & $\sqrt{ }$ & $\sqrt{ }$ \\
\hline Edinburgh Postnatal Depression Scale & & & & $\sqrt{ }$ & & & & \\
\hline State-Trait Anxiety Inventory for Adults & & & & $\sqrt{ }$ & & & & \\
\hline Six Months Postpartum Questionnaire & & & & & & & & $\sqrt{ }$ \\
\hline
\end{tabular}

of anxiety (20 items) and tendency or trait anxiety (20 items)

g) Six Months Postpartum Questionnaire - collects data on sources of infant feeding advice, participation in other relevant parenting support programmes, adequacy and main source of household financial resources since the infant's birth, and maternal receipt of federal Employment Insurance maternity benefits (yes/no)

Table 1 shows the timing of data collection for each study instrument. Prenatal data will be collected in the third trimester. The 2-week and 1-month data will be collected within 7 days before or after the exact time point, and the other data will be collected within 14 days before or after the exact time point with the exception of the two maternal mental health forms (EPDS and STAI). These may be collected up to 3 months postpartum. The greater time window is to enable completion of these forms in person due to the sensitive nature of the content and the higher rates of depression and anxiety among newcomer mothers reported in the literature [43]. Research staff will arrange to meet study participants at their home or a preferred location in their community if they are unable to attend either the $\mathrm{HB}$ or GST program to complete these forms. A safety protocol will be followed to ensure appropriate support and follow-up for any participants with scores $>21$ on the EPDS, or who indicate any thoughts on self-harm in response to EPDS question 10.

The Infant Feeding Questionnaire has been professionally translated into Chinese and back-translated by a second translator. We will utilize published Spanish translations of the Infant Feeding Intentions [38] and Prenatal Breastfeeding Self-Efficacy Scale [44]. Translations into Chinese, Spanish and Portuguese were accessed from a compilation of validation studies for the EPDS [45] and were provided by CLD for the Breastfeeding Self-Efficacy Scale-Short Form and by MindGarden, Inc. for the STAI. Other study forms have been translated into Chinese by our $\mathrm{Re}$ search Assistant or will be orally translated at the time of data collection by professional interpreters.

In order to monitor feasibility of implementation delivery, the Research Coordinator will track the number of clients eligible for the intervention, the number who participate (defined as receiving at least one IBCLC visit), and the specific services provided (number of home visits and breast pump provision, if applicable). Reasons for clients not accessing the intervention will also be recorded. The IBCLCs will complete a data collection form after each home visit, recording the assessment components covered, concerns identified and services provided. These data will be analysed to determine reach, uptake and fidelity of the intervention delivery.

\section{Data management}

Data collection forms will be identified by participant number only. Participant contact information and the code linking participant numbers to names will be stored in password-protected files on an encrypted USB key accessible only to AM. These files will be destroyed on completion of study publications. Completed data collection forms will be stored in a locked filing cabinet in a locked office and will be retained for 7 years postpublication as per University of Toronto procedures. Anonymized data will be entered into a spreadsheet stored on a different encrypted USB key before being transferred to statistical software for analysis.

\section{Statistical analysis}

Descriptive statistics will be generated for all variables. Primary and secondary outcome measures will be compared between the pre- and post-intervention groups using t-tests for continuous variables and chisquare statistics for categorical variables. Sub-group analysis will be performed to examine differences in 


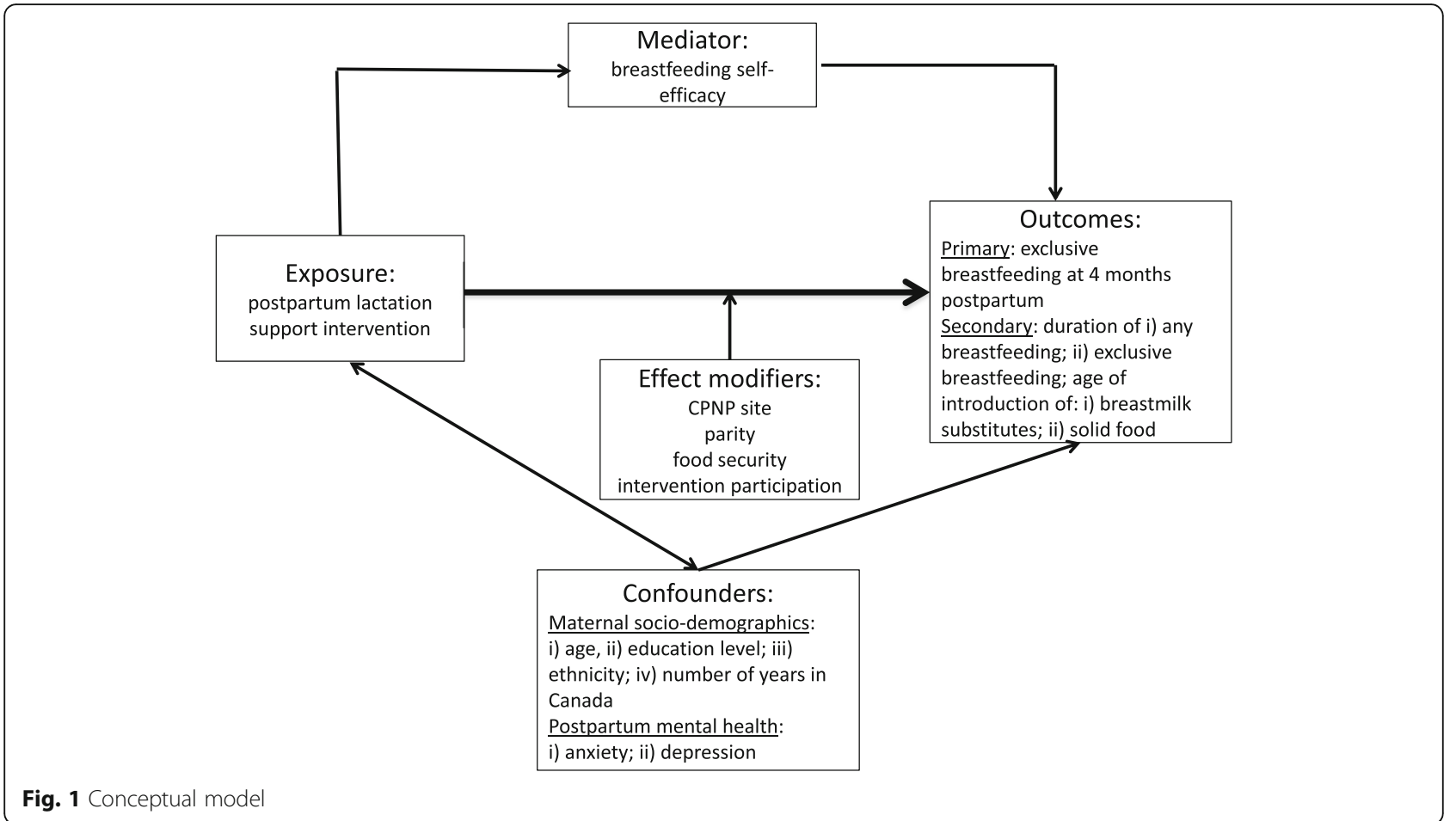

outcomes based on the effect modifiers (program site, parity, food security status and intervention participation) as shown in the conceptual model (Fig. 1). Logistic regression analysis will be used to determine the relationship between breastfeeding self-efficacy scores at 2 months postpartum and breastfeeding practices at 4 and 6 months postpartum, controlling for confounders. All statistical analysis will be performed using IBM SPSS Statistics for Windows, version 26 (IBM Corp., Armonk, N.Y., USA).

\section{Discussion}

The CPNP is a national initiative which aims to promote and support breastfeeding but currently lacks a formal framework for integrating postpartum lactation support, a key component of enabling women to achieve their breastfeeding goals. This pre/post intervention study aims to examine the effects on breastfeeding practices of pro-actively offering inhome lactation support to vulnerable women through two CPNP sites in Toronto. Feasibility will also be examined through analysis of monitoring data to determine the reach, uptake and fidelity of the intervention. We hypothesize that CPNP clients with access to our lactation support intervention will have higher breastfeeding self-efficacy scores at 2 months postpartum and be more likely to exclusively breastfeed their infants at 4 months postpartum compared with those in the pre-intervention group.

To date there has been limited evaluation of breastfeeding outcomes among CPNP participants. This study will contribute data on infant feeding practices at seven time points in the first 6 months postpartum, and will examine a range of covariates including measures of maternal mental health, household food insecurity and socio-demographic characteristics. Strengths of this research include the prospective data collection, theorybased intervention and inclusion of multiple covariates. $\mathrm{AM}$ and the Research Coordinator will attend the weekly program at both CPNP sites, and the Mandarin speaking Research Assistant will attend the weekly GST program. This embeddedness within the programs will contribute to the building of trust and rapport with participants, and is expected to result in high enrollment and retention rates [46]. However, the participant characteristics and infant feeding practices of women registered at the two study sites in Toronto may not be representative of CPNPs across the country and the pre/post study design is not as strong as a randomized trial for determining effectiveness. This research is therefore one step in the process of generating the evidence for appropriate models of integrating lactation support with the CPNP and thereby increasing breastfeeding, health and developmental outcomes for vulnerable infants in Canada. 


\section{Abbreviations}

CPNP: Canada Prenatal Nutrition Program; EPDS: Edinburgh Postnatal Depression Scale; GST: Great Start Together (perinatal program); HB: Healthy Beginnings (perinatal program); IBCLC: International Board Certified Lactation Consultant; 5Ps: Parkdale Parents' Primary Prevention Project; STAI: State-Trait Anxiety Inventory

\section{Acknowledgements}

We thank Stephanie Zhang, Research Coordinator, and Yiqin Mao, Research Assistant, for their key contributions to the implementation of this research, and all staff and partners of the GST and HB programs for supporting the integration of this study within their program activities.

\section{Authors' contributions}

AM, JF, DLO and DWS led the design of the study with contributions from all authors. SS, BU and YN facilitated the selection of study sites, and YN, ER and $C R$ managed the integration of research activities into the GST and HB programs. AM, DLO and DWS are leading the research implementation. AM drafted this protocol manuscript with input from all co-authors. All authors read and approved the final manuscript.

\section{Funding}

This work is supported by a Canadian Institutes of Health Research Doctoral Research Award (\#GSD-157928) to AM, and by The Sprott Foundation and the Joannah and Brian Lawson Centre for Child Nutrition. The funders had no role in the study design and will not be involved in data analysis, interpretation or drafting of the manuscripts.

\section{Availability of data and materials}

Not applicable.

\section{Declarations}

\section{Ethics approval and consent to participate}

Ethics approval has been obtained for this study from the Office of Research Ethics at the University of Toronto (protocol \#35845) and the Toronto Public Health Research Ethics Board (protocol \#2018-11). Study aims, procedures, risks and benefits including procedures to protect participant privacy and confidentiality will be explained at the time of recruitment, and all participants will provide written informed consent.

\section{Consent for publication}

Not applicable.

\section{Competing interests}

The authors declare that they have no competing interests.

\section{Author details}

${ }^{1}$ Nutritional Sciences, Temerty Faculty of Medicine, University of Toronto, Toronto, Ontario, Canada. ${ }^{2}$ Translational Medicine Program, The Hospital for Sick Children, Toronto, Ontario, Canada. ${ }^{3}$ Health Promotion and Community Engagement, Parkdale-Queen West Community Health Centre, Toronto, Ontario, Canada. ${ }^{4}$ The Stop Community Food Centre, Toronto, Ontario, Canada. ${ }^{5}$ Tamarack Institute, Waterloo, Ontario, Canada. ${ }^{6}$ Dalla Lana School of Public Health, University of Toronto, Toronto, Ontario, Canada.

${ }^{7}$ Lawrence-Bloomberg Faculty of Nursing, University of Toronto, Toronto, Ontario, Canada. ${ }^{8}$ Anthropology, Faculty of Arts and Sciences, University of Toronto, Toronto, Ontario, Canada.

Received: 24 August 2020 Accepted: 8 June 2021

Published online: 02 July 2021

\section{References}

1. Victora CG, Bahl R, Barros AJD, França GVA, Horton S, Krasevec J, et al. Breastfeeding in the 21st century: epidemiology, mechanisms, and lifelong effect. Lancet. 2016;387(10017):475-90. https://doi.org/10.1016/S0140-6736(1 5)01024-7

2. Health Canada, Canadian Paediatric Society, Dietitians of Canada and Breastfeeding Committee for Canada. Nutrition for healthy term infants: recommendations from birth to six months. Ottawa: Health Canada; 2015. https://www.canada.ca/en/health-canada/services/food-nutrition/healthy-ea ting/infant-feeding/nutrition-healthy-term-infants-recommendations-birthsix-months.html. Accessed 20 Nov 2017.

3. World Health Organization. Global strategy for infant and young child feeding. Geneva: WHO; 2003. https://www.who.int/nutrition/publications/ gs_infant_feeding_text_eng.pdf. Accessed 9 Nov 2016.

4. Development Initiatives. 2020 Global Nutrition Report: action on equity to end malnutrition. Bristol: Development Initiatives; 2020. https://globa Inutritionreport.org/reports/2020-global-nutrition-report/. Accessed 16 Jul 2020.

5. Public Health Agency of Canada. Breastfeeding. In: Family-centred maternity and newborn care: national guidelines. Ottawa: PHAC; 2019. https://www.ca nada.ca/en/public-health/services/publications/healthy-living/maternitynewborn-care-guidelines-chapter-6.html. Accessed 21 Jul 2020

6. Azad MB, Vehling L, Chan D, Klopp A, Nickel NC, McGavock JM, et al. Infant feeding and weight gain: separating breast milk from breastfeeding and formula from food. Pediatrics. 2018;142(4):e20181092. https://doi.org/10.1542/peds.2018-1092

7. Jessri M, Farmer AP, Maximova K, Willows ND, Bell RC, APrON Study Team. Predictors of exclusive breastfeeding: observations from the Alberta pregnancy outcomes and nutrition (APrON) study. BMC Pediatr. 2013;13(1): 77. https://doi.org/10.1186/1471-2431-13-77.

8. Al-Sahab B, Lanes A, Feldman M, Tamim H. Prevalence and predictors of 6month exclusive breastfeeding among Canadian women: a national survey. BMC Pediatr. 2010;10(1):20. https://doi.org/10.1186/1471-2431-10-20.

9. Gionet L. Health at a glance: breastfeeding trends in Canada. Ottawa: Statistics Canada; 2013. https://www150.statcan.gc.ca/n1/pub/82-624-x/2013 001/article/11879-eng.pdf. Accessed 27 Oct 2017

10. Orr SK, Dachner N, Frank L, Tarasuk V. Relation between household food insecurity and breastfeeding in Canada. CMAJ. 2018;190(11):E312-E9. https://doi.org/10.1503/cmaj.170880.

11. Orr SK, Tarasuk V. Breastfeeding and maternal physical and mental health among food insecure families with infants in Canada. FASEB J. 2016; 30(Suppl. 1):Ib435.

12. Best Start Resource Centre. Populations with lower rates of breastfeeding: a summary of findings. Toronto: BSRC; 2014. https://resources.beststart.org/ wp-content/uploads/2018/12/B09-E.pdf. Accessed 13 Jul 2017.

13. Dennis CL, Shiri R, Brown HK, Santos HP Jr, Schmied V, Falah-Hassani K. Breastfeeding rates in immigrant and non-immigrant women: a systematic review and meta-analysis. Matern Child Nutr. 2019;15(3):e12809. https://doi. org/10.1111/mcn.12809.

14. Health Canada. Duration of exclusive breastfeeding in Canada: key statistics and graphics (2009-2010). Ottawa: Health Canada; 2012. http://www.hc-sc. gc.ca/fn-an/surveill/nutrition/commun/prenatal/exclusive-exclusif-eng.php. Accessed 8 Feb 2017

15. Brown CRL, Dodds L, Legge A, Bryanton J, Semenic S. Factors influencing the reasons why mothers stop breastfeeding. Can J Public Health. 2014; 105(3):e179-e85. https://doi.org/10.17269/cjph.105.4244.

16. Li R, Fein SB, Chen J, Grummer-Strawn LM. Why mothers stop breastfeeding: mothers' self-reported reasons for stopping breastfeeding during the first year. Pediatrics. 2008;122(Suppl. 2):S69-76. https://doi.org/1 0.1542/peds.2008-1315i.

17. Odom EC, RLi R, Scanlon KS, Perrine CG, Grummer-Strawn L. Reasons for earlier than desired cessation of breastfeeding. Pediatrics. 2013;131(3):e726e32. https://doi.org/10.1542/peds.2012-1295.

18. Pérez-Escamilla R, Buccini GS, Segura-Pérez S, Piwoz E. Perspective: should exclusive breastfeeding still be recommended for 6 months? Adv Nutr. 2019:10(6):931-43. https://doi.org/10.1093/advances/nmz039.

19. Dennis $\mathrm{CL}$. Theoretical underpinnings of breastfeeding confidence: a selfefficacy framework. J Hum Lact. 1999;15(3):195-201. https://doi.org/10.1177/ 089033449901500303

20. Brockway M, Benzies K, Hayden KA. Interventions to improve breastfeeding self-efficacy and resultant breastfeeding rates: a systematic review and meta-analysis. J Hum Lact. 2017;33(3):486-99. https://doi.org/10.1177/ 0890334417707957

21. de Jager E, Skouteris H, Broadbent J, Amir L, Mellor K. Psychosocial correlates of exclusive breastfeeding: a systematic review. Midwifery. 2013; 29(5):506-18. https://doi.org/10.1016/j.midw.2012.04.009.

22. Chetwynd EM, Wasser HM, Poole C. Breastfeeding support interventions by International Board Certified Lactation Consultants: a systematic review and meta-analysis. J Hum Lact. 2019:35(3):424-40. https://doi.org/10.1177/ 0890334419851482.

23. Kim SK, Park S, Oh J, Kim J, Ahn S. Interventions promoting exclusive breastfeeding up to six months after birth: a systematic review and meta- 
analysis of randomized controlled trials. Int J Nurs Stud. 2018 Apr;80:94-105. https://doi.org/10.1016/j.jinurstu.2018.01.004.

24. McFadden A, Gavine A, Renfrew MJ, Wade A, Buchanan P, Taylor JL, et al. Support for healthy breastfeeding mothers with healthy term babies. Cochrane Database Syst Rev. 2017;2:CD001141.

25. Patel $\mathrm{S}$, Patel $\mathrm{S}$. The effectiveness of lactation consultants and lactation counselors on breastfeeding outcomes. J Hum Lact. 2016;32(3):530-41. https://doi.org/10.1177/0890334415618668.

26. Francis J, Mildon A, Stewart S, Underhill B, Tarasuk V, Di Ruggiero E, et al. Vulnerable mothers' experiences breastfeeding with an enhanced community lactation support program. Matern Child Nutr. 2020;16(3): e12957. https://doi.org/10.1111/mcn.12957.

27. Public Health Agency of Canada. Canada Prenatal Nutrition Program (CPNP). Ottawa: PHAC; 2020. https://www.canada.ca/en/public-health/services/hea Ith-promotion/childhood-adolescence/programs-initiatives/canada-prenatalnutrition-program-cpnp.html. Accessed 19 Oct 2020

28. Health Canada and the Public Health Agency of Canada. Evaluation of the Community Action Program for Children, Canada Prenatal Nutrition Program and associated activities 2010-2011 to 2014-2015. Ottawa: PHAC 2016. https://www.canada.ca/en/public-health/corporate/transparency/ corporate-management-reporting/evaluation/2010-2011-2014-2015-evalua tion-community-action-program-children-canada-prenatal-nutrition-progra m-associated-activities.html. Accessed 5 Jul 2017

29. Public Health Agency of Canada. Protecting, promoting and supporting breastfeeding: a practical workbook for community-based programs, 2nd edition. Ottawa: PHAC; 2014. https://www.canada.ca/en/public-health/ services/health-promotion/childhood-adolescence/publications/protectingpromoting-supporting-breastfeeding.html. Accessed 5 May 2017

30. Statistics Canada. Table 11-10-0241-01. Low income cut-offs (LICOs) before and after tax by community size and family size, in current dollars. Ottawa: Statistics Canada; 2020. https://www150.statcan.gc.ca/t1/tbl1/en/tv.a ction?pid=1110024101. Accessed 16 Jul 2020

31. Labiner-Wolfe J, Fein SB, Shealy KR, Wang C. Prevalence of breast milk expression and associated factors. Pediatrics. 2008;122(Suppl 2):S63-8. https://doi.org/10.1542/peds.2008-1315h.

32. Win NN, Binns CW, Zhao Y, Scott JA, Oddy WH. Breastfeeding duration in mothers who express breast milk: a cohort study. Int Breastfeed J. 2006;1(1): 28. https://doi.org/10.1186/1746-4358-1-28.

33. Bordelon C, Wood T, Stallworth K. Clinician's guide to supporting women with breast milk pumping. Nurs Womens Health. 2019;23(5):440-9. https:// doi.org/10.1016/j.nwh.2019.07.003.

34. Meier PP, Patel AL, Hoban R, Engstrom JL. Which breast pump for which mother: an evidence-based approach to individualizing breast pump technology. J Perinatol. 2016;36(7):493-9. https://doi.org/10.1038/jp.2016.14.

35. O'Connor DL, Khan S, Weishuhn K, Vaughan J, Jefferies A, Campbell DM, et al. Growth and nutrient intakes of human milk-fed preterm infants provided with extra energy and nutrients after hospital discharge. Pediatrics. 2008;121 (4):766-76. https://doi.org/10.1542/peds.2007-0054

36. Dennis CL. The breastfeeding self-efficacy scale: psychometric assessment of the short form. J Obstet Gynecol Neonatal Nurs. 2003;32(6):734-44. https:// doi.org/10.1177/0884217503258459.

37. Statistics Canada. Employment insurance coverage survey - 2018. Ottawa: Statistics Canada; 2018. https://www23.statcan.gc.ca/imdb/p3Instr. pl?Function=assemblelnstr\&lang=en\&ltem_Id=494316\#qb367084. Accessed 7 Jan 2019

38. Nommsen-Rivers LA, Dewey KG. Development and validation of the infant feeding intentions scale. Matern Child Health J. 2009;13(3):334-42. https:// doi.org/10.1007/s10995-008-0356-y.

39. Wells KJ, Thompson NJ, Kloeblen-Tarver AS. Development and psychometric testing of the prenatal breastfeeding self-efficacy scale. Am J Health Behav. 2006;30(2):177-87. https://doi.org/10.5993/AJHB.30.2.7.

40. Health Canada. Canadian Community Health Survey cycle 2.2, nutrition (2004) - income-related household food security in Canada. Ottawa: Health Canada; 2007. https://www.canada.ca/content/dam/hc-sc/migration/hc-sc/ fn-an/alt_formats/hpfb-dgpsa/pdf/surveill/income_food_sec-sec_alim-eng. pdf. Accessed 31 Oct 2017.

41. Cox JL, Holden JM, Sagovsky R. Detection of postnatal depression. Development of the 10-item Edinburgh Postnatal Depression Scale. Br J Psychiatry. 1987;150(6):782-6. https://doi.org/10.1192/bjp.150.6.782.

42. Spielberger C, Gorsuch R, Lushene R, Vagg P, Jacobs G. Manual for the State-Trait Anxiety Inventory. Palo Alto: Consulting Psychologists Press; 1983.
43. Falah-Hassani K, Shiri R, Vigod S, Dennis CL. Prevalence of postpartum depression among immigrant women: a systematic review and metaanalysis. J Psychiatr Res. 2015;70:67-82. https://doi.org/10.1016/j.jpsychires.2 015.08.010.

44. Pineiro-Albero RM, Ramos-Pichardo JD, Oliver-Roig A, Velandrino-Nicolas A, Richart-Martinez M, Garcia-de-Leon-Gonzalez R, et al. The Spanish version of the prenatal breastfeeding self-efficacy scale: reliability and validity assessment. Int J Nurs Stud. 2013;50(10):1385-90. https://doi.org/10.1016/j. ijnurstu.2012.12.010

45. Department of Health, Government of Western Australia. Edinburgh Postnatal Depression Scale (EPDS): Translated versions - validated. Perth: State Perinatal Mental Health Reference Group; 2006. https:/www.mcpa pformoms.org/Docs/Edinburgh\%20Depression\%20Scale\%20Translated\%2 OGovernment\%20of\%20Western\%20Australia\%20Department\%20of\%20Hea Ith.pdf. Accessed 6 Dec 2018

46. Barnett J, Aguilar S, Brittner M, Bonuck K. Recruiting and retaining lowincome, multi-ethnic women into randomized controlled trials: successful strategies and staffing. Contemp Clin Trials. 2012;33(5):925-32. https://doi. org/10.1016/j.cct.2012.06.005

\section{Publisher's Note}

Springer Nature remains neutral with regard to jurisdictional claims in published maps and institutional affiliations.

Ready to submit your research? Choose BMC and benefit from:

- fast, convenient online submission

- thorough peer review by experienced researchers in your field

- rapid publication on acceptance

- support for research data, including large and complex data types

- gold Open Access which fosters wider collaboration and increased citations

- maximum visibility for your research: over $100 \mathrm{M}$ website views per year

At $\mathrm{BMC}$, research is always in progress.

Learn more biomedcentral.com/submissions 